Article

\title{
Prediction of Bone Metastasis in Breast Cancer Based on Minimal Driver Gene Set in Gene Dependency Network
}

\author{
Jia-Nuo Li ${ }^{\dagger}$, Rui Zhong ${ }^{\dagger}$ and Xiong-Hui Zhou * \\ Hubei Key Laboratory of Agricultural Bioinformatics, College of Informatics, Huazhong Agricultural University, \\ Wuhan 430070, China; ljn2126212@163.com (J.-N.L.); zr295110971@163.com (R.Z.) \\ * Correspondence: zhouxionghui@mail.hzau.edu.cn; Tel.: +86-27-8728-0877 \\ + These authors contribute equally to this work.
}

Received: 27 March 2019; Accepted: 14 June 2019; Published: 17 June 2019

check for updates

\begin{abstract}
Bone is the most frequent organ for breast cancer metastasis, and thus it is essential to predict the bone metastasis of breast cancer. In our work, we constructed a gene dependency network based on the hypothesis that the relation between one gene and the risk of bone metastasis might be affected by another gene. Then, based on the structure controllability theory, we mined the driver gene set which can control the whole network in the gene dependency network, and the signature genes were selected from them. Survival analysis showed that the signature could distinguish the bone metastasis risks of cancer patients in the test data set and independent data set. Besides, we used the signature genes to construct a centroid classifier. The results showed that our method is effective and performed better than published methods.
\end{abstract}

Keywords: breast cancer; bone metastasis; gene dependency network; structure controllability theory; driver gene set

\section{Introduction}

Breast cancer, one of the most frequent tumors, seriously affects human health [1,2]. Metastasis is the main cause of death in breast cancer patients, and bone is the most frequently metastatic organ [3]. Therefore, it is necessary to explore the mechanism of bone metastasis in breast cancer [4], and the research predicting bone metastasis in breast cancer is crucial [5].

At present, several works have attempted to explain the mechanism of bone metastasis in breast cancer by using molecular biology methods [6-8]. However, only a few studies have mined the prognostic makers and predicted the bone metastasis in breast cancer. Previously, we identified the key genes of breast cancer by identifying dysregulated pathways in the bone metastasis of breast cancer. The prediction model that we constructed to predict the risk of bone metastasis of breast cancer-the dysregulated pathway-based prediction model (DPBM) [9]—achieved a certain predictive effect, but the performance is still unsatisfactory.

Since cancer is a complex disease, a biological network that can reveal the gene regulatory relations in cancer becomes an effective tool [10]. In our previous work, we proposed a method to infer the gene dependency network which could uncover the gene regulatory relations during cancer metastasis, based on the hypothesis that the influence of one gene on the prognosis may depend on the other genes [11], and this network can be used to mine essential genes in the prognosis of cancer. Therefore, it may be promising to construct a gene dependency network in the bone metastasis of breast cancer.

In recent years, some exciting developments about the control of biological systems have been made [12], such as the structural controllability theory, referring to a system which could be controlled 
from one state to another by controlling several nodes (i.e., genes) in the system (biological network) [13]. Therefore, it is to use the gene dependency network in the bone metastasis of breast cancer as the biological system and identify the driver gene set based on the structural controllable theory. In addition, the driver gene set may be helpful for the construction of a prediction model.

In this work, we integrated the gene expression profiles and clinical information of 855 breast cancer patients to construct the gene dependency network, which could uncover the gene dependency relations in the process of bone metastasis in breast cancer [14]. Then, combined with the structural controllability theory we mined the driver nodes in the network as candidate features, based on which the gene signature was selected and a centroid classifier was constructed. We also validated our method by comparing it with other published methods.

\section{Materials and Methods}

\subsection{Data Sets and Pre-Processing}

We downloaded the gene expression profiles of breast cancer patients along with the clinical information from the UNC microarray database [15]. The downloaded data consisted of four microarray data sets: GSE2034 [16], GSE2603 [17], GSE12276 [18], and NKI295 [19]. The raw data was processed and the clinical information of the patients (time of bone-metastasis and status of bone metastasis) was extracted as in the previous work [15], and statistical "batch-effect correction" procedures were already performed on the data. For the sake of comparing the results with other methods, we adopted the same strategy as the DPBM to divide the data set into training, test, and independent data sets. GSE2034 was used as the independent data set. In the remaining samples, 380 samples were selected as the training set and the other 189 samples were used as the test data set. For constructing the gene dependency network, the expression levels of all the genes and the clinical information were binarized. For a gene, the expression level of a patient was set as 0 if it was not more than the median of the gene's expression levels in all the patients. Otherwise, it was set as 1 . As to the clinical information, in usual work, the 5-years rule is extensively recognized as the limitation in prognosis or metastasis risk prediction in breast cancer $[20,21]$, and bone is the most common metastatic organ of breast cancer which occupied a large number of samples, so we chose 5 years as the threshold to determine the risk of bone metastasis of breast cancer patients. If a patient had bone metastasis within 5 years, they were set as high-risk (1); if they had bone-metastasis-free survival for more than 5 years, they were set in the low-risk group (0); otherwise, the patient was abandoned.

Furthermore, the human protein-protein interactions from HIPPIE (Human Integrated Protein-Protein Interaction rEference) [22] were also downloaded.

\subsection{Construction of Gene Dependency Network}

In our previous work, we proposed a method to infer the gene dependency network which could uncover the gene regulatory relations during cancer metastasis [11], based on the hypothesis that the influence of one gene on the metastasis may depend on another gene. Here, we applied it to infer the gene dependency network in the process of bone metastasis of breast cancer. Specifically, for two genes $A$ and $B$, if the influence of gene $A$ on phenotype (bone metastasis risk) is modulated by gene $B$, and we believe that gene $A$ depends on gene $B$. Conditional mutual information (CMI) was applied to calculate the gene dependency relation between gene $A$ and gene $B$, and a permutation test was performed to evaluate the significance of the gene dependency relation. The pipeline to construct the gene dependency network is shown as follows:

(1) In order to reduce the false discovery rate, only the gene pairs involved in the protein-protein interaction network were used as candidates. Here, all the gene pairs in HIPPIE [22] were set as candidates for gene dependency pairs.

(2) For each candidate gene dependency pair (e.g., gene $A$ and gene $B$ ), we set the expression levels of gene $A$ and gene $B$ and the bone-metastasis risks of all the patients as a triple. The triple was 
sorted according to the expression level of gene $B$ in ascending order. Then, the conditional mutual information was calculated by using Equation (1):

$$
\mathrm{CMI}(\text { Gene } A ; \text { risk } \mid \text { GeneB })=I_{\text {high }}(\text { GeneA }, \text { risk })-I_{\text {low }}(\text { Gene } A, \text { risk })
$$

where $I_{\text {high }}($ Gene $A$, risk) is the mutual information of gene $A$ and the bone-metastasis risks of $35 \%$ cancer patients whose expression levels of gene $B$ are high. $I_{\text {low }}$ (GeneA, risk) is the mutual information of gene $A$ and the bone-metastasis risks of $35 \%$ of cancer patients whose expression levels of gene $B$ are low.

(3) A permutation test was proposed to calculate the $p$-value of the conditional mutual information for each gene pair. First, we randomly permuted the expression levels of gene $B$. Secondly, a random CMI was calculated for gene $A$ and gene $B$ using the method described in step (2). Thirdly, the random permutation was repeated 1000 times and 1000 random CMIs were obtained, and the $p$-value of the CMI was calculated based on the 1000 random CMIs. Finally, all the significant gene dependency pairs $(p$-value $<0.05)$ were combined as the gene dependency network.

\subsection{Structural Controllability of Networks}

According to the controllability of complex networks [13], the non-linear processes (and linear processes) can be studied using the canonical linear and time-invariant dynamics.

The system described by Equation (2):

$$
\frac{d x(t)}{d t}=A x(t)+B u(t)
$$

is controllable if it can be driven from any initial state to any desired final state in finite time, which is possible if and only if the controllability matrix $N \times N M$ has full rank. That is, the matrix $C$ in Equation (3) follows Equation (4).

$$
\begin{gathered}
C=\left(\mathrm{B}, \mathrm{AB}, \mathrm{A}^{2} B, \ldots, A^{N-1} B\right) \\
\operatorname{rank}(C)=N
\end{gathered}
$$

Full controllability can be also posed by identifying the minimum number of driver nodes such that Equation (4) is satisfied [13]. However, it is a computationally prohibitive task for large networks [15], so it is to apply in practical problems. If it is possible to satisfy Equation (4) by selecting non-zero weights in $A$ and $B$, the system is structurally controllable [23]. As the calculation process of structural controllability does not need to measure the link weights, it is acceptable in computational complexity. Furthermore, structural controllability can be solved by identifying the maximum matching in directed networks. Therefore, in this work, we applied the structure controllability theory to study the controllability of the bone metastasis system in breast cancer and identify the driver nodes in the system (direct network) as biomarker candidates.

\subsection{Selecting the Driver Gene Sets by Controllability}

Based on the hypothesis that the gene dependency network in the bone metastasis of breast cancer can reveal the biological mechanism in bone metastasis, the driver nodes that could structurally control the network may be crucial in the biological process of bone metastasis. Therefore, we identified these driver nodes as biomarker candidates.

As we described above, structural controllability can be solved by identifying the maximum matching in directed networks - that is, the minimal driver set that can drive all the nodes in the network. Here, the maximum matching algorithm [24] was applied to identify the minimal driver set in the gene dependency network, and these nodes were set as the driver nodes. However, as we 
know, the solutions of maximum matching in directed networks are not unique, especially in complex networks. In this work, we randomly permuted the index of nodes (keeping the dependency relations among the nodes) and ran this program for 500 times to get the frequencies of the occurrence of genes in the minimal driver set, and we chose the genes with the frequency of 500 as the candidate genes. That is, the nodes (genes) in all the driver sets were set as feature (biomarker) candidates.

In order to construct the classifier to predict the bone metastasis of cancer patients, the features should be different in the two patient groups (i.e., high-risk and low-risk groups). Therefore, the feature candidates were further selected by the $t$-test, so as to select the genes which were differentially expressed in the two groups (in the training data set). As a result, the driver nodes with $p$-values less than 0.002 were set as features.

\subsection{Construction of the Classifier}

In this work, we applied the centroid classifier to construct the prediction model. The first reason we chose it is that the centroid classifier is suitable for microarray data, which has the character of large feature size and few samples [25]. Secondly, the centroid classifier does not need to offer the extra adjustment of the parameter. Besides, the centroid classifier is difficult to overfit [26]. Supposing the number of bone metastases in breast cancer patients is $N$, and $s_{1}, s_{n}$, each $s_{i}$ represents the gene expression level vector from a patient. $s_{i}$ contains the $M$ dimensions of the measurement value of the patient. Making a short explain about the $s_{i}(i, j)$, it represents the expression level of the $i$-th feature (gene) in the $j$-th patient. Above that, we denoted the $n^{+}$patients belonging to the positive class (with the label 1) and the $n^{-}$patients belonging to negative class (with the label 0) with Equation (5):

$$
N=n^{+}+n^{-}
$$

Then, we could calculate the gene expression level centroid (mean value) vector with $M$ dimensions separated by the class according to Equations (6) and (7) as follows:

$$
\begin{aligned}
& \vec{C}^{+}=\frac{1}{n^{+}} \sum_{S_{j} \in \text { Class }+} S_{j}(i, j) \\
& \vec{C}^{-}=\frac{1}{n^{-}} \sum_{S_{j} \in \text { Class }-} S_{j}(i, j)
\end{aligned}
$$

In Equation (8)

$$
\vec{C}=\left(\vec{C}^{+}+\vec{C}^{-}\right) / 2
$$

$\vec{C}$ means the mean vector of the centroids, and in Equation (9)

$$
\vec{w}=\vec{C}^{+}-\vec{C}^{-}
$$

$\vec{w}$ is the weight vector of the $M$ features. If the feature vector of a sample is $s=\left(d_{1}, \ldots, d_{M}\right)$, we can appraise the samples by the following Equation (10):

$$
\gamma=\langle\vec{s}=-\vec{C}, \vec{w}\rangle
$$

If $\gamma>0$, then $\widetilde{s}$ is assigned to a positive class; otherwise, it is assigned to a negative class. 


\subsection{Tools and Package}

The functions of the selected driver genes were annotated by GSEA (Gene Set Enrichment Analysis) [27]. The network was analyzed and visualized by using Cytoscape 3.6.1 and the survival analysis was performed using the R package "survival".

\section{Results}

\subsection{Basic Information of the Gene Dependency Network}

We constructed the gene dependency network in the process of bone metastasis in breast cancer using conditional mutual information. It contained 10,163 gene pairs (Table S1) among 5380 genes (Figure S1). As a result, both in-degree and out-degree followed to the power law distribution, with the R-square values 0.946 and 0.942 , and the correlation coefficients were 0.942 and 0.985 . The average number of neighbors, both in-degree and out-degree, were 1.889. The results suggest that our gene dependency network is scale free and coincident with the typical characteristics of biological network.

In addition, we also found some remarkable pairs in the gene dependency network related to breast cancer and metastasis. For instance, MYC deregulation is conductive to development and progression in breast cancer [28]. Wnt signaling in breast cancer is important [29]. It has been reported that MYC can activate WNT in breast cancer [30], and in our work, Wnt1 was significantly dependent on MYC (with $p$-value of 0.002). As we know, TP53 is a famous gene in breast cancer which can influence cancer prognosis [31]. Migration in breast cancer can be suppressed by targeting MYO10 [32]. In our gene dependency network, TP53 and MYO10 had a significant dependency relation (with $p$-value of 0.013), and according to the literature, the expression of MYO10 is relevant to the expression of TP53 in breast tumors [33]. Furthermore, the BRCA1 gene is also crucial in breast cancer, which has an impact on breast cancer risk [34]. EZH2 is a marker of aggressive breast cancer [35]. It has been found that the downregulation of EZH2 decreases invasive breast cancer and requires BRCA1 [36]. In our network, EZH2 was dependent on BRCA1 with a $p$-value of 0.009. Therefore, our gene dependency network was reliable and could be used to study the bone metastasis of breast cancer.

\subsection{Functional Annotation of the Driver Nodes}

As we described above, structural controllability can be solved by identifying the maximum matching in directed networks, and the minimal driver set driving all the nodes in our gene dependency network may be important in the bone-metastasis process of breast cancer. Here, we selected driver nodes as feature candidates to construct the prediction model. The number of nodes in the minimal driver set in our network was 2483 , which is about $46 \%$ of all the nodes. In a previous work [13], it was proved that about $80 \%$ of the nodes in biological networks should be controlled to satisfy the structural controllability, which may indicate that the gene dependency is easier to control.

Although the driver nodes in the network may be essential, there are two problems here hindering the application of the minimal driver gene set in feature selection. First of all, the size of the driver set is too large. As described above, almost $80 \%$ of all the nodes in biological networks are driver nodes. Even in our gene dependency network, 2483 genes were driver nodes. In addition, the solutions of the maximum matching are not unique, especially in complex networks. To solve these problems, we randomly permuted the index of nodes (keeping the dependency relations among the nodes) and ran this program for 500 times to get the frequency of the occurrence of genes in the minimal driver set, and we chose the genes with the frequency of 500 as the candidate genes. As a result, 1864 genes from the gene dependency network were selected as feature candidates (Table S2).

We also used GSEA [27] to investigate the function of the feature candidates. The top 10 KEGG (Kyoto Encyclopedia of Genes and Genomes) pathways are listed in Table 1.

The MAPK signaling pathway is the most significant pathway in breast cancer metastasis. Meanwhile, through suppression of the MAPK signaling pathway, metastasis of breast cancer cells can be reduced [37]. In addition, it has been reported that HGFK1 could inhibit bone metastasis in breast 
cancer through the TAK1/p38 MAPK signaling pathway [38]. Furthermore, the feature candidates are significantly enriched by pathways in cancer, with a $p$-value of $2.82 \times 10^{-13}$. The feature candidates were significantly involved in the pathway of focal adhesion $\left(p\right.$-value $\left.=4.05 \times 10^{-11}\right)$, and it has been reported that focal adhesion kinase is a prominent determinant in the metastasis of breast cancer [39], has an effect on bone tumors, and may be crucial in bone metastasis [40]. The cytokine-cytokine receptor interaction is also significant $\left(p\right.$-value $\left.=8.22 \times 10^{-11}\right)$, and cytokines are relevant to the microenvironment of bone metastasis in breast cancer [41].

In conclusion, the genes which are frequently involved in the minimal driver gene sets are related to the metastasis, even bone metastasis in breast cancer.

Table 1. Functional annotation of the driver gene set.

\begin{tabular}{ccc}
\hline KEGG Gene Set Name & $p$-Value & FDR q-Value \\
\hline MAPK signaling pathway & $7.31 \times 10^{-19}$ & $1.36 \times 10^{-16}$ \\
Neuroactive ligand-receptor interaction & $1.12 \times 10^{-15}$ & $1.04 \times 10^{-13}$ \\
Pathways in cancer & $2.82 \times 10^{-13}$ & $1.75 \times 10^{-11}$ \\
Focal adhesion & $4.05 \times 10^{-11}$ & $1.88 \times 10^{-9}$ \\
Cytokine-cytokine receptor interaction & $8.22 \times 10^{-11}$ & $3.06 \times 10^{-9}$ \\
Regulation of actin cytoskeleton & $2.72 \times 10^{-10}$ & $8.43 \times 10^{-9}$ \\
SNARE (SNAP Receptor) interactions in vesicular transport & $1.62 \times 10^{-9}$ & $4.29 \times 10^{-8}$ \\
Complement and coagulation cascades & $1.26 \times 10^{-8}$ & $2.92 \times 10^{-7}$ \\
Purine metabolism & $3.08 \times 10^{-8}$ & $6.36 \times 10^{-7}$ \\
Spliceosome & $5.07 \times 10^{-8}$ & $9.42 \times 10^{-7}$ \\
\hline
\end{tabular}

KEGG: Kyoto Encyclopedia of Genes and Genomes; FDR: False discovery rate.

\subsection{The Selected Features}

Based on the feature candidates (the genes involved in all the minimal driver gene sets), we selected 51 signature genes (Table 2 ) with $p$-values ( $t$-test) less than 0.002 in order to find the differential expression genes.

In the signature, almost all the genes were related to cancer, and many genes were associated with bone metastasis of breast cancer. FSTL1 promotes bone metastasis by causing immune dysfunction [42]. It has been reported that Wnt signaling is related to bone metastasis in breast cancer [43] and that DIXDC1 can promote the metastasis of cancer cells by activating the Wnt signaling pathway [44]. Breast cancer patients with bone metastasis presented with reduced expression of NDRG1 and their survival was also influenced by NDRG1 expression [45]. Experiments have shown that a group of genes including MLPH can predict bone metastasis in breast cancer well [46]. CRIM1 is a potential risk factor of cancer that regulates the migration of cancer cells [47]. RANBP10 may help inhibit the growth of breast cancer cells [48]. The expression of RXRA also plays a key role in breast cancer [49].

In all, the genes in the signature were involved the minimal driver gene set, and they were distinguished in high-risk and low-risk groups. In addition, most of these genes are indeed related to the metastasis in breast cancer. Therefore, our gene signatures may be good features for classification in the bone metastasis of breast cancer. 
Table 2. The signature genes in our work.

\begin{tabular}{|c|c|c|c|}
\hline Gene Id & Gene Name & Frequency & $p$-Value \\
\hline 85458 & DIXDC1 & 500 & $1.68192 \times 10^{-6}$ \\
\hline 29068 & ZBTB44 & 500 & $3.29096 \times 10^{-6}$ \\
\hline 51232 & CRIM1 & 500 & $1.46030 \mathrm{e} \times 10^{-5}$ \\
\hline 9986 & RCE1 & 500 & $2.12898 \times 10^{-5}$ \\
\hline 56888 & KCMF1 & 500 & $4.00030 \times 10^{-5}$ \\
\hline 1456 & CSNK1G3 & 500 & $4.58237 \times 10^{-5}$ \\
\hline 6256 & RXRA & 500 & $7.63144 \times 10^{-5}$ \\
\hline 55343 & SLC35C1 & 500 & 0.00012 \\
\hline 55520 & ELAC1 & 500 & 0.00012 \\
\hline 55081 & IFT57 & 500 & 0.00012 \\
\hline 57610 & RANBP10 & 500 & 0.00018 \\
\hline 5877 & RABIF & 500 & 0.00018 \\
\hline 25839 & COG4 & 500 & 0.00019 \\
\hline 23261 & CAMTA1 & 500 & 0.00020 \\
\hline 3009 & HIST1H1B & 500 & 0.00020 \\
\hline 3092 & HIP1 & 500 & 0.00026 \\
\hline 246243 & RNASEH1 & 500 & 0.00030 \\
\hline 3104 & ZBTB48 & 500 & 0.00031 \\
\hline 10342 & TFG & 500 & 0.00032 \\
\hline 6282 & S100A11 & 500 & 0.00033 \\
\hline 10462 & CLEC10A & 500 & 0.00033 \\
\hline 51199 & NIN & 500 & 0.00041 \\
\hline 10531 & PITRM1 & 500 & 0.00048 \\
\hline 9856 & KIAA0319 & 500 & 0.00049 \\
\hline 11167 & FSTL1 & 500 & 0.00049 \\
\hline 3993 & LLGL2 & 500 & 0.00052 \\
\hline 56729 & RETN & 500 & 0.00054 \\
\hline 51514 & DTL & 500 & 0.00054 \\
\hline 9202 & ZMYM4 & 500 & 0.00058 \\
\hline 51302 & CYP39A1 & 500 & 0.00065 \\
\hline 9971 & NR1H4 & 500 & 0.00067 \\
\hline 79083 & MLPH & 500 & 0.00073 \\
\hline 65082 & VPS33A & 500 & 0.00075 \\
\hline 10179 & RBM7 & 500 & 0.00078 \\
\hline 55794 & DDX28 & 500 & 0.00082 \\
\hline 57405 & SPC25 & 500 & 0.00089 \\
\hline 51659 & GINS2 & 500 & 0.00089 \\
\hline 1852 & DUSP9 & 500 & 0.00092 \\
\hline 57017 & COQ9 & 500 & 0.00096 \\
\hline 10397 & NDRG1 & 500 & 0.00098 \\
\hline 9911 & TMCC2 & 500 & 0.00128 \\
\hline 55095 & SAMD4B & 500 & 0.00137 \\
\hline 23649 & POLA2 & 500 & 0.00143 \\
\hline 10615 & SPAG5 & 500 & 0.00143 \\
\hline 7134 & TNNC1 & 500 & 0.00145 \\
\hline 7083 & TK1 & 500 & 0.00146 \\
\hline 9442 & MED27 & 500 & 0.00151 \\
\hline 8449 & DHX16 & 500 & 0.00171 \\
\hline 8817 & FGF18 & 500 & 0.00176 \\
\hline 483 & ATP1B3 & 500 & 0.00179 \\
\hline 2175 & FANCA & 500 & 0.00190 \\
\hline
\end{tabular}

The frequency of a gene is the times involved in the minimal driver gene sets and the $p$-values were calculated based on $t$-test. 


\subsection{Survival Analysis of Breast Cancer Patients}

In order to predict the risk of bone metastasis in breast cancer patients, we divided the patients in the training data set into two groups (i.e., high-risk and low-risk groups). Using the genes in the signature as features, we constructed a centroid classifier to predict the bone metastasis of cancer patients based on the training data set. Then, the classifier was applied in the test data set and independent data set. We performed survival analysis of the patients in both the test data set and independent data set respectively to validate our classifier.

In the test data set, 71 patients were predicted as high-risk patients, and 98 patients were predicted as low-risk patients. Survival analysis showed that the bone-metastasis risks of the patients in the two groups were indeed different, with a $p$-value of 0.007 (Figure 1).

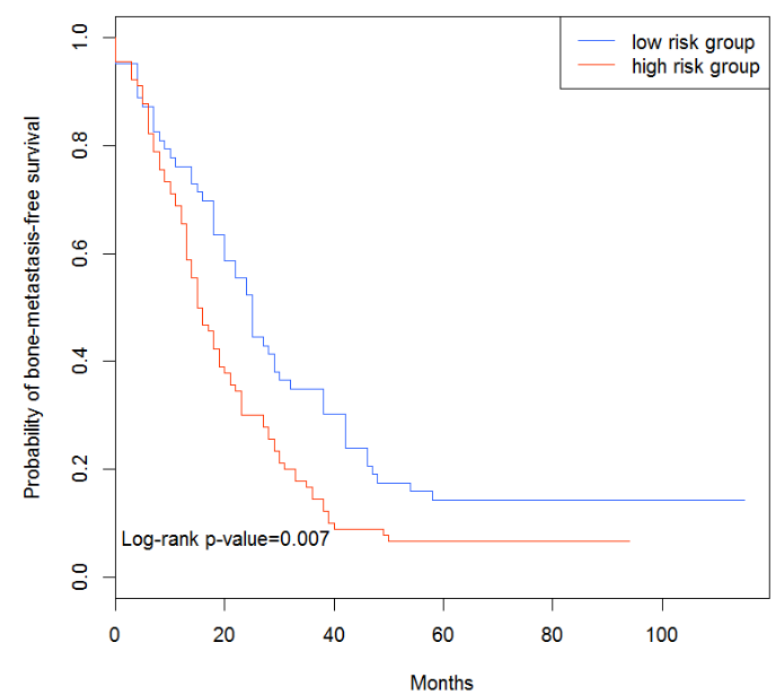

Figure 1. Survival analysis of test data set.

Among the 286 patients in the independent test set, 117 were classified as high-risk patients, and 169 patients were classified as low-risk patients. The $p$-value of the log-rank test of the patients in the two groups was 0.002 (Figure 2), indicating that the low-risk patients predicted by our method had longer survival time (bone-metastasis-free survival) than the high-risk ones.

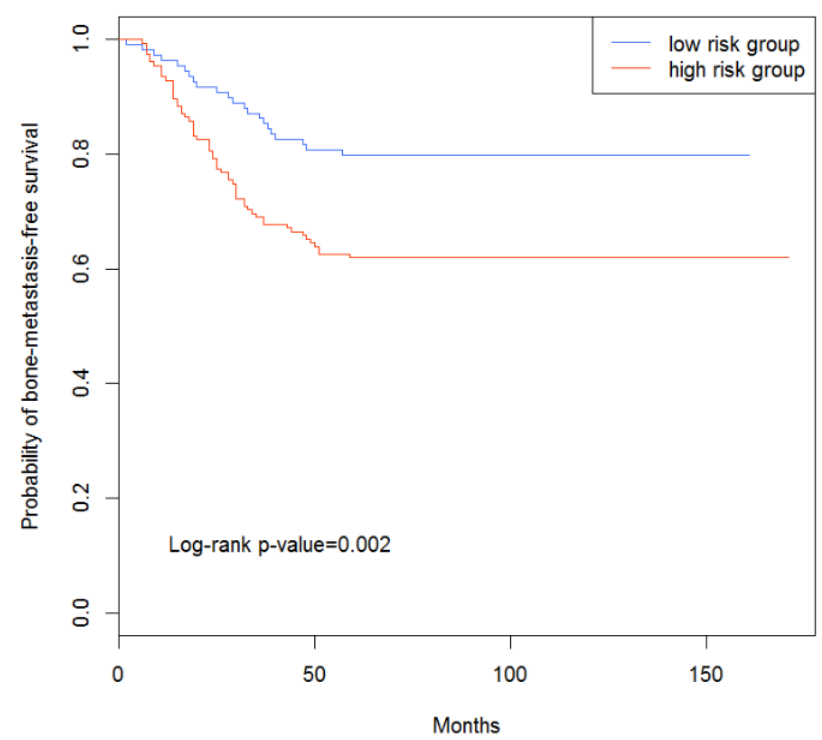

Figure 2. Survival analysis of the independent data set. 
From the results, it could be demonstrated that our classifier could distinguish the bone-metastasis risks in the test and independent data sets, indicating that the features (i.e., genes) we selected could indeed play a key role in the bone metastasis of breast cancer.

\subsection{Comparison with Other Methods}

In the previous work, the dysregulated pathway-based prediction model (DPBM) [11] was proposed to predict the bone metastasis of breast cancer patients. In addition, two other classifiers, support vector machine (SVM) and shrunken centroids classifier (SCC), using the same genes in DPBM as features, were also compared with the DPBM in the original work. As our work used the same data sets as the DPBM, we compared our centroid classifier with the DPBM, SVM, and SCC to validate the performance of our work.

The AUC (area under the curve) of the four classifiers in the test data set and independent data set are shown in Figure 3 (the details of the performance are shown in Table S3). As a result, our centroid classifier could achieve AUCs of 0.65 and 0.66 in the two data sets, respectively, while the best AUC of all the other methods was about 0.60 . That is, our centroid classifier outperformed other methods and our method dramatically improved the predictive performance in bone metastasis.

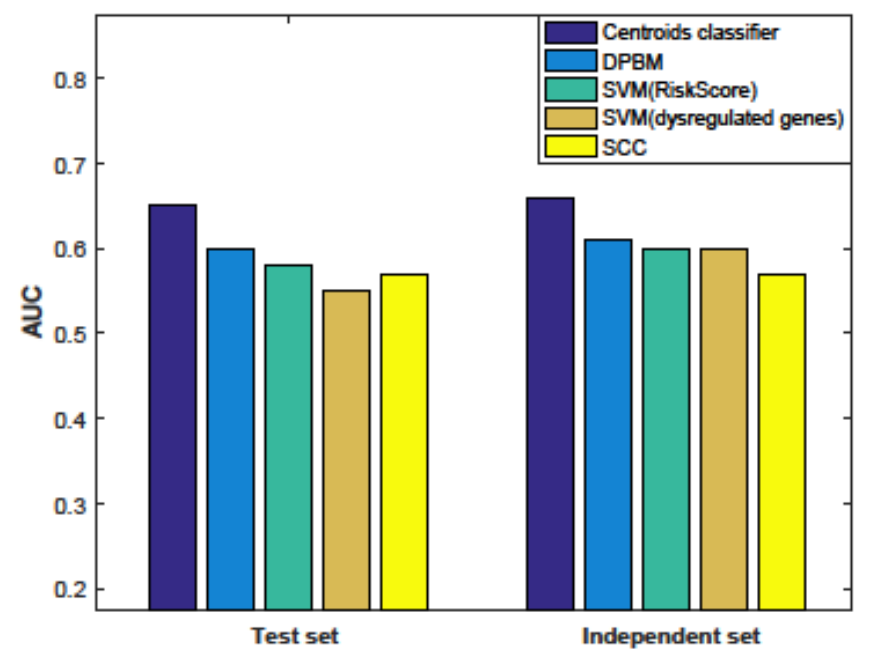

Figure 3. Comparing the performance of our method with published methods. AUC: area under the curve; DPBM: dysregulated pathway-based prediction model. SVM: Support vector machine; SCC: Shrunken centroids classifier.

\subsection{Evaluation the Significance of Our Method}

We made predictions based on our signature genes in other organ metastases (lung, liver, brain, and any organs) to verify the specificity of bone metastasis. We calculated the AUC and accuracy of five organs in the training, test, and independent data sets. The results are shown in Table S4 and Figure 4 . We found that our signature had very limited predictive ability for each of the other organs. In addition, the performance of metastasis prediction in any organs (the traditional overall survival or distant-metastasis-free survival) was good, but slightly worse than bone-metastasis prediction. This is because most of the organ metastases are bone metastases. This illustrates that our signature genes have specificity for bone metastasis. Moreover, it has been reported that the status of a single gene (e.g., estrogen receptor (ER)) can distinguish the bone-metastasis risk in breast cancer [50], so we also compared the performance of ER status and our method in evaluating the risk of bone metastasis of breast cancer. Since ER status listed in the data set is not a continuous risk score but a discrete status, we used the MCC (Matthews correlation coefficient), which is a classification evaluation index for unbalanced data sets, to evaluate the performance of our signature and ER status (Table 3). The results show that our method was better than 
the use of single genes (e.g., ER status) in classification. Therefore, our gene signature performed better than a traditional clinical index (i.e., ER status) and had specificity for bone metastasis.

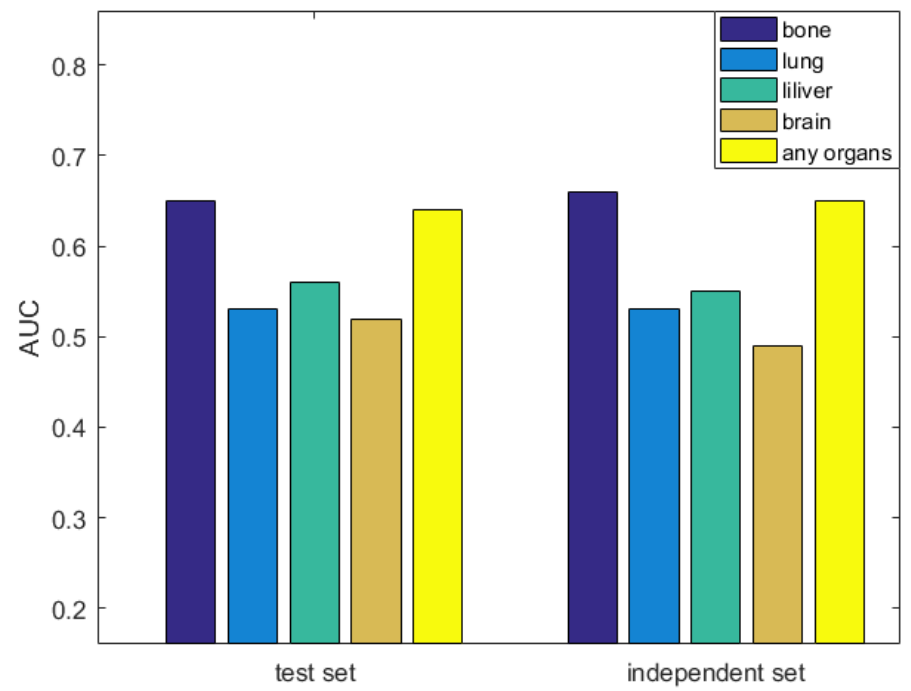

Figure 4. Comparing the performance of bone metastasis with other organ metastases.

Table 3. Comparing results with estrogen receptor (ER) status.

\begin{tabular}{cccc}
\hline & Training Data Set & Test Data Set & Independent Data Set \\
\hline Centroids classifier & 0.22 & 0.15 & 0.20 \\
ER status & 0.18 & 0.16 & 0.07 \\
\hline
\end{tabular}

\section{Discussion}

Predicting the bone metastasis of breast cancer patients is still a challenge. In this work, we proposed a new method based on the minimal driver gene set in a gene dependency network to predict the risk of bone metastasis of breast cancer.

First of all, we used a merged data set which contained the transcriptome data and clinical information of 855 breast cancer patients. Then, the conditional mutual information was applied to infer the gene dependency pairs in the network for which the mutual information between one gene and the bone-metastasis risk was significantly dependent on the other genes. All the significant gene pairs were constituted as a gene dependency network, and the network was scale free. Besides, case study and function annotation confirmed that the network could reveal the gene dependency relation in the process of bone metastasis. Structure controllability theory states that a system (e.g., biological network) can be controlled by controlling the nodes in the minimal driver gene set. Therefore, the driver nodes in the gene dependency network may be biomarker candidates. However, the size of the minimal driver gene set for the driver sets is too large (In our work, 2483 genes were in each minimal driver gene set). In addition, the solutions of the maximum matching are not unique, especially in complex networks. To solve these problems, we calculated multiple minimal driver gene sets and selected the genes which were involved in all the minimal driver gene sets as feature candidates. Function annotation illustrated that these genes were related to bone metastasis in breast cancer. Based on the feature candidates, 51 genes which were differentially expressed were selected as features, and a centroid classifier was constructed. Our method was validated by survival analysis and through comparison with other methods, and the results showed that our method dramatically improved the predictive performance in bone metastasis.

In conclusion, we provided a new method to study the bone metastasis in breast cancer. The method uncovered the regulation mechanism in bone metastasis by constructing a gene dependency network. A new feature selection method based on the minimal driver gene set in structure controllability theory 
was proposed to select the key genes. Finally, the prediction model was constructed by using the essential genes as features. In fact, our method can also be used to study other biological problems, as long as there are enough genotypic and phenotypic data. We will validate our method in other biological problems in our future work.

Supplementary Materials: The following are available online at http://www.mdpi.com/2073-4425/10/6/466/s1, Figure S1: The gene dependency network, Table S1: The gene dependency network of bone metastasis in breast cancer, Table S2: The feature candidates, Table S3: Comparing results with other methods, Table S4: Comparing results with other organs.

Author Contributions: X.-H.Z. designed research; X.-H.Z. and J.-N.L. performed the research; X.-H.Z., R.Z., and J.-N.L. wrote the paper; J.-N.L. analyzed the data. All authors revised the manuscript.

Funding: This work was supported by the National Natural Science Foundation of China (61602201), the Fundamental Research Funds for the Central Universities (2662018PY023).

Conflicts of Interest: The authors declare no conflict of interest.

\section{References}

1. Hedayat, A.; Parvin, H.; Abazar, R.; Seyyed Hamid, M.; Shokrallah, M.; Mojtaba, S.; Mohsen, B.; Ali, A. Relationships between Breast Cancer and Common Non-Communicable Disease Risk Factors: An Ecological Study. Asian Pac. J. Cancer Prev. APJCP 2013, 14, 5123-5125.

2. Feig, S.A. 4-8 A Basal Epithelial Phenotype Is More Frequent in Interval Breast Cancers Compared with Screen Detected Tumors. Breast Dis. Year Book Q. 2006, 16, 337-338. [CrossRef]

3. Thomas, L.; Amanda, J.; Akeila, B.; Nadia, R.; Soraya, S.; Berta Martin, A.; Angels, S.; Alain, B.; Jean-Marc, G.; Enrico, R. A six-gene signature predicting breast cancer lung metastasis. Cancer Res. 2008, 68, 6092.

4. Müller, A.; Homey, B.; Soto, H.; Ge, N.; Catron, D.; Buchanan, M.E.; Mcclanahan, T.; Murphy, E.; Yuan, W.; Wagner, S.N. Involvement of chemokine receptors in breast cancer metastasis. Nature 2001, 410, 50-56. [CrossRef] [PubMed]

5. Nogi, H.; Takeyama, H.; Uchida, K.; Agata, T.; Horiguchi-Yamada, J.; Yamada, H. Detection of MUC1 and keratin 19 mRNAs in the bone marrow by quantitative RT-PCR predicts the risk of distant metastasis in breast cancer patients. Breast Cancer 2003, 10, 74-81. [CrossRef] [PubMed]

6. Kingsley, L.A.; Fournier, P.G.; Chirgwin, J.M.; Guise, T.A. Molecular biology of bone metastasis. Mol. Cancer Ther. 2007, 6, 2609-2617. [CrossRef]

7. Akhtari, M.; Mansuri, J.; Newman, K.A.; Guise, T.; Seth, P. Biology of breast cancer bone metastasis. Cancer Biol. Ther. 2008, 7, 3-9. [CrossRef] [PubMed]

8. Woelfle, U.; Cloos, J.; Sauter, G.; Riethdorf, L.; Jänicke, F.; Van, D.P.; Brakenhoff, R.; Pantel, K. Molecular signature associated with bone marrow micrometastasis in human breast cancer. Cancer Res. 2003, 63, 5679-5684.

9. Zhou, X.; Liu, J. A computational model to predict bone metastasis in breast cancer by integrating the dysregulated pathways. BMC Cancer 2014, 14, 618. [CrossRef]

10. Khalid, S.; Hanif, R.; Tareen, S.H.K.; Siddiqa, A.; Bibi, Z.; Ahmad, J. Formal modeling and analysis of ER- $\alpha$ associated Biological Regulatory Network in breast cancer. PeerJ 2016, 4, e2542. [CrossRef]

11. Zhou, X.; Liu, J. Inferring gene dependency network specific to phenotypic alteration based on gene expression data and clinical information of breast cancer. PLoS ONE 2014, 9, e92023. [CrossRef] [PubMed]

12. Li, M.; Gao, H.; Wang, J.; Wu, F. Control principles for complex biological networks. Brief. Bioinform. 2018. [CrossRef] [PubMed]

13. Yang-Yu, L.; Jean-Jacques, S.; Albert-László, B. Controllability of complex networks. Nature 2011, 473, 167.

14. Motakis, E.; Ivshina, A.V.; Kuznetsov, V.A. Data-driven approach to predict survival of cancer patients: Estimation of microarray genes' prediction significance by Cox proportional hazard regression model. IEEE Eng. Med. Biol. Mag. 2009, 28, 58-66. [CrossRef] [PubMed]

15. Harrell, J.C.; Aleix, P.; Parker, J.S.; Cheng, F.; Xiaping, H.; Lisa, C.; Carey, A.; Matthew, E.; Perou, C.M. Genomic analysis identifies unique signatures predictive of brain, lung, and liver relapse. Breast Cancer Res. Treat. 2012, 132, 523-535.

16. Hayes, D.F. 2-20 Gene-Expression Profiles to Predict Distant Metastasis of Lymph-Node-Negative Primary Breast Cancer. Lancet 2006, 17, 154-155. [CrossRef] 
17. Minn, A.J.; Gupta, G.P.; Siegel, P.M.; Bos, P.D.; Weiping, S.; Giri, D.D.; Agnes, V.; Olshen, A.B.; Gerald, W.L.; Joan, M. Genes that mediate breast cancer metastasis to lung. Nature 2005, 436, 518-524. [CrossRef]

18. Bos, P.D.; Zhang, X.H.F.; Nadal, C.; Shu, W.; Gomis, R.R.; Nguyen, D.X.; Minn, A.J.; van de Vijver, M.J.; Gerald, W.L.; Foekens, J.A.; et al. Genes that mediate breast cancer metastasis to the brain. Nature 2009, 459, 1005-1009. [CrossRef]

19. Aziz, N.A.A.; Mokhtar, N.M.; Harun, R.; Mollah, M.M.H.; Rose, I.M.; Sagap, I.; Tamil, A.M.; Wan, Z.W.N.; Jamal, R. A 19-Gene expression signature as a predictor of survival in colorectal cancer. BMC Med. Genomics 2016, 9, 58.

20. Tomich, P.L.; Helgeson, V.S.J.P.-O. Five years later: A cross-sectional comparison of breast cancer survivors with healthy women. Psycho-Oncol. J. Psychol. Soc. Behav. Dimens. Cancer 2010, 11, 154-169. [CrossRef]

21. Snyder, C.F.; Frick, K.D.; Peairs, K.S.; Kantsiper, M.E.; Herbert, R.J.; Blackford, A.L.; Wolff, A.C.; Earle, C.C. Comparing care for breast cancer survivors to non-cancer controls: A five-year longitudinal study. J. Gen. Intern. Med. 2009, 24, 469-474. [CrossRef] [PubMed]

22. Schaefer, M.H.; Fontaine, J.; Vinayagam, A.; Porras, P.; Wanker, E.E.; Andradenavarro, M.A. HIPPIE: Integrating protein interaction networks with experiment based quality scores. PLoS ONE 2012, 7, e31826. [CrossRef] [PubMed]

23. Shields, R.; Pearson, J. Structural controllability of multiinput linear systems. IEEE Trans. Autom. Control 1976, 21, 203-212. [CrossRef]

24. Galil, Z. Efficient algorithms for finding maximal matching in graphs. In Colloquium on Trees in Algebra and Programming; Springer: Berlin/Heidelberg, Germany, 1983; Volume 159, pp. 90-113. [CrossRef]

25. Bedo, J.; Sanderson, C.; Kowalczyk, A. An efficient alternative to SVM based recursive feature elimination with applications in natural language processing and bioinformatics. In Proceedings of the Australian Joint Conference on Artificial Intelligence: Advances in Artificial Intelligence, Hobart, Australia, 4-8 December 2006.

26. Abraham, G.; Kowalczyk, A.; Loi, S.; Haviv, I.; Zobel, J. Prediction of breast cancer prognosis using gene set statistics provides signature stability and biological context. BMC Bioinform. 2010, 11, 277. [CrossRef] [PubMed]

27. Subramanian, A.; Tamayo, P.; Mootha, V.K.; Mukherjee, S.; Ebert, B.L.; Gillette, M.A.; Paulovich, A.G.; Pomeroy, S.L.; Golub, T.R.; Lander, E.S. Gene set enrichment analysis: A knowledge-based approach for interpreting genome-wide expression profiles. Proc. Natl. Acad. Sci. USA 2005, 102, 15545-15550. [CrossRef] [PubMed]

28. Jinhua, X.; Yinghua, C.; Olopade, O.I. MYC and Breast Cancer. Genes Cancer 2010, 1, 629-640.

29. Brown, A.M. Wnt signaling in breast cancer: Have we come full circle? Breast Cancer Res. 2001, 3, 351-355. [CrossRef] [PubMed]

30. Cowling, V.; Cole, M. Turning the tables: Myc activates Wnt in breast cancer. Cell Cycle 2007, 6, $2625-2627$. [CrossRef]

31. Anne-Lise, B.R.D. TP53 and breast cancer. Hum. Mutat. 2003, 21, 292-300.

32. Chen, C.P.; Sun, Z.L.; Lu, X.; Wu, W.X.; Guo, W.L.; Lu, J.J.; Han, C.; Huang, J.Q.; Fang, Y. miR-340 suppresses cell migration and invasion by targeting MYO10 in breast cancer. Oncol. Rep. 2015, 35, 709. [CrossRef]

33. Antti, A.; Riina, K.; Elina, M.; Pegah, R.; Gunilla, H.G.S.; Harri, S.; Miller, B.W.; Morton, J.P.; Elmar, B.; Pekka, T. Mutant p53-associated myosin-X upregulation promotes breast cancer invasion and metastasis. J. Clin. Investig. 2014, 124, 1069.

34. Mary-Claire, K.; Marks, J.H.; Mandell, J.B. Breast and ovarian cancer risks due to inherited mutations in BRCA1 and BRCA2. Science 2003, 302, 643-646.

35. Kleer, C.G.; Qi, C.; Sooryanarayana, V.; Ronglai, S.; Ichiro, O.; Tomlins, S.A.; Debashis, G.; Sewalt, R.G.A.B.; Otte, A.P.; Hayes, D.F. EZH2 is a marker of aggressive breast cancer and promotes neoplastic transformation of breast epithelial cells. Proc. Natl. Acad. Sci. USA 2003, 100, 11606-11611. [CrossRef] [PubMed]

36. Gonzalez, M.E.; Li, X.; Toy, K.; DuPrie, M.; Ventura, A.C.; Banerjee, M.; Ljungman, M.; Merajver, S.D.; Kleer, C.G. Downregulation of EZH2 decreases growth of estrogen receptor-negative invasive breast carcinoma and requires BRCA1. Oncogene 2009, 28, 843-853. [CrossRef] [PubMed]

37. Bangxing, H.; Haiyan, L.; Mingjun, Z.; Jingda, X.; Yong, L.; Yuhuan, Z.; Jianfei, Q.; Chang, J.T.; Jing, Y.; Qing, Y. p38 MAPK inhibits breast cancer metastasis through regulation of stromal expansion. Int. J. Cancer 2015, 136, 34-43. 
38. Yao, Y.; Fang, Z.P.; Chen, H.; Yue, L.; Min, D.L.; Tang, L.N.; Yu, W.X.; Kung, H.F.; Lin, M.C.; Shen, Z. HGFK1 inhibits bone metastasis in breast cancer through the TAK1/p38 MAPK signaling pathway. Cancer Gene Ther. 2012, 19, 601-608. [CrossRef] [PubMed]

39. Luo, M.; Guan, J.L. Focal adhesion kinase: A prominent determinant in breast cancer initiation, progression and metastasis. Cancer Lett. 2010, 289, 127-139. [CrossRef] [PubMed]

40. Bagi, C.M.; Roberts, G.W.; Andresen, C.J. Dual focal adhesion kinase/Pyk2 inhibitor has positive effects on bone tumors: Implications for bone metastases. Cancer 2010, 112, 2313-2321. [CrossRef]

41. Bussard, K.M.; Venzon, D.J.; Mastro, A.M. Osteoblasts are a major source of inflammatory cytokines in the tumor microenvironment of bone metastatic breast cancer. J. Cell. Biochem. 2010, 111, 1138-1148. [CrossRef]

42. Kudo-Saito, C. FSTL1 promotes bone metastasis by causing immune dysfunction. OncoImmunology 2013, 2, e26528. [CrossRef]

43. Johnson, R.W.; Merkel, A.R.; Page, J.M.; Ruppender, N.S.; Guelcher, S.A.; Sterling, J.A.J.C.; Metastasis, E. Wnt signaling induces gene expression of factors associated with bone destruction in lung and breast cancer. Clin. Exp. Metastasis 2014, 31, 945-959. [CrossRef] [PubMed]

44. Tan, C.; Qiao, F.; Wei, P.; Chi, Y.; Wang, W.; Ni, S.; Wang, Q.; Chen, T.; Sheng, W.; Du, X. DIXDC1 activates the Wnt signaling pathway and promotes gastric cancer cell invasion and metastasis. Mol. Carcinog. 2016, 55, 397-408. [CrossRef] [PubMed]

45. Sucharita, B.; Pai, S.K.; Shigeru, H.; Sadahiro, H.; Yukio, T.; Ken, S.; David, P.; Therese, C.; Misako, W.; Gross, S.C. Role of the putative tumor metastasis suppressor gene Drg-1 in breast cancer progression. Oncogene 2004, 23, 5675-5681.

46. Cosphiadi, I.; Atmakusumah, T.D.; Siregar, N.C.; Abdulmuthalib; Harahap, A.; Mansyur, M.J.C.B.C. Bone Metastasis in Advanced Breast Cancer: Analysis of Gene Expression Microarray. Clin. Breast Cancer 2018, 18, e1117-e1122. [CrossRef] [PubMed]

47. Zeng, H.; Zhang, Y.; Yi, Q.; Wu, Y.; Wan, R.; Tang, L. CRIM1, a newfound cancer-related player, regulates the adhesion and migration of lung cancer cells. Growth Factors 2015, 33, 384. [CrossRef]

48. Naoki, H.; Tsuyoshi, Y.; Ryoichi, Y.; Yoshihisa, N.; Hiroshi, I. RanBP10 acts as a novel coactivator for the androgen receptor. Biochem. Biophys. Res. Commun. 2008, 368, 121-125.

49. Yee, L.D.; Sabourin, C.L.; Liu, L.; Li, H.M.; Smith, P.J.; Seewaldt, V.; Kniss, D.A. Peroxisome proliferator-activated receptor $\gamma$ activation in human breast cancer. Int. J. Oncol. 1999, 15, 967. [CrossRef]

50. Zhang, X.H.F.; Wang, Q.; Gerald, W.; Hudis, C.A.; Norton, L.; Smid, M.; Foekens, J.A.; Massagué, J. Latent Bone Metastasis in Breast Cancer Tied to Src-Dependent Survival Signals. Cancer Cell 2009, 16, 67-78. [CrossRef]

(C) 2019 by the authors. Licensee MDPI, Basel, Switzerland. This article is an open access article distributed under the terms and conditions of the Creative Commons Attribution (CC BY) license (http://creativecommons.org/licenses/by/4.0/). 